\title{
Analysis on the Composition and Breakthrough of Modern Chinese Dialect Grammar
}

\author{
Yu Wenjing \\ Dongchang College, Liaocheng University, Shandong, China 252000
}

Keywords: modern Chinese, dialect grammar, composition, breakthrough

Abstract: Professor Zong Shouyun's "Zhangjiakou Jinyu Grammar Study" (Commercial Press, April 2018) is a new achievement of the author's grammar research, and is also a representative work of domestic scholars in the recent exploration of Jin language grammar. This book consists of an introduction and four chapters of the text, which involves many aspects of the study of grammar in Jin dynasty. It has made new breakthroughs in the in-depth analysis of dialect grammar and the revealing of modern Chinese grammar rules. For the current dialect grammar and even the entire Chinese grammar study, there are important implications in both theory and practice.

\section{Introduction}

For a long time, people have focused on the dialectical analysis of the phonetic and semantic aspects of dialects, and basically formed a research method based on diachronic investigation. For the dialect grammar, there is still to be explored in the research paradigm of research methods. "Zhangjiakou Jinyu Grammar Study" draws on the subjective theory and semantic grammar of contemporary language, and makes a useful attempt in this respect. This article explains and discusses the author's own experience and practice by expounding the author's analysis and tasting of the book.

\section{A new perspective on the composition of modern language grammar}

\subsection{The composition of modern Chinese grammar}

The Zhangjiakou Jin Language Grammar Study selected a unique and novel perspective to study dialects. This is the form. Starting from the form, taking into account both meaning and function, this is the basic path of modern Chinese grammar research. On the other hand, how to describe Chinese grammatical forms and their marks in detail, and then to make scientific induction from the perspective of morphological types and grammatical categories, is undoubtedly one of the keys to further breakthroughs in Chinese grammar from theory to practice. For a long time, researchers have done a lot of work on the grammatical forms and descriptions of modern Chinese Mandarin. In comparison, the exploration of modern Chinese dialects is obviously weak. 


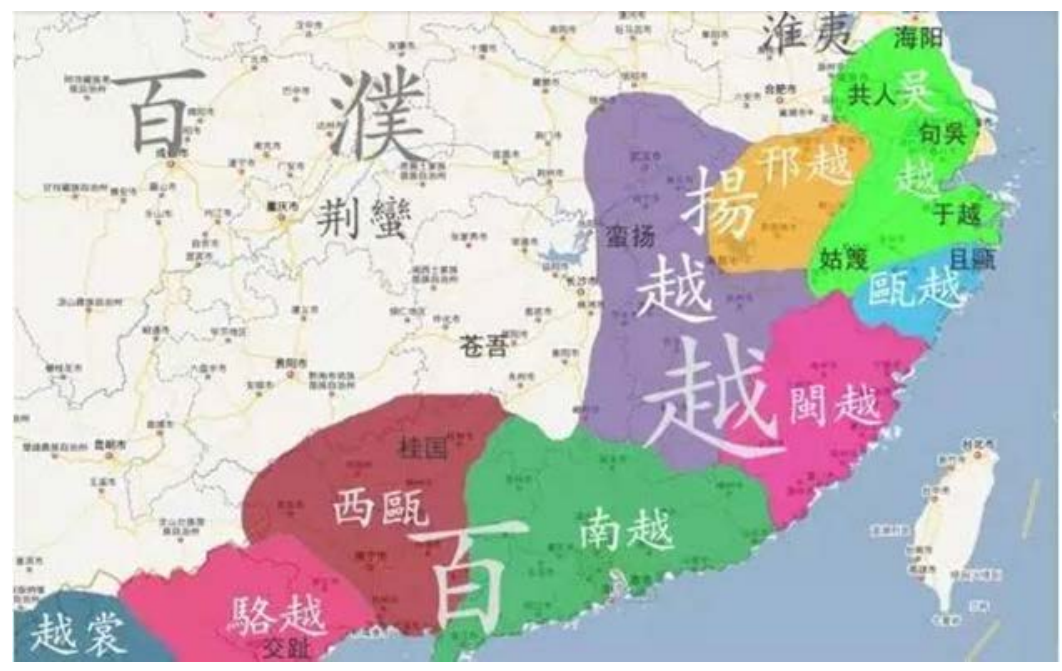

Figure 1: The distribution of grammatical roots in contemporary Chinese dialects

As shown in Figure 1 above, "Zhangjiakou Jinyu Grammar Study" selects the Jin language familiar to the author. The first chapter begins with the picture and dialect form, analyzes the tail, overlap, inflection and derivation of Zhangjiakou Jin language. The phonetic, semantic and grammatical features of the "sub-variable rhyme" are described in the "sub-variable rhyme table" of the basic vowel of the urban rhyme of the Zhuolu County. This is undoubtedly of great value in revealing the grammatical features of Jin dialect and even the entire Chinese dialect. For another example, through the description of single words and the basic law induction, the author finds that "shaming into two readings" is a manifestation of the inflected form of Jin language, which has implications for understanding Chinese form from a broader perspective. And about the phenomenon of derivation,

\subsection{A new perspective of modern Chinese grammar}

The author cites the same root two-tone state words coexisting in the 93 groups of Lushan dialects. Based on the detailed corpus, the semantic grammatical essence of the phenomenon of "derived" is further revealed from the perspective of meaning and function. In addition, the author also focuses on the asymmetry of the color meaning of the Jin language state words, including 20 innocents and 62 innocents, and analyzes the basic rationale of the asymmetry from the theoretical level. The above research results undoubtedly use the perspective of form, combined with meaning and function, with detailed description and refined analysis, to discover a series of grammatical phenomena that were originally ignored by researchers. These provide us with an in-depth understanding of Jin language and other modern times. The nature and laws of Chinese dialect grammar provide a new path.

\section{A new breakthrough method in modern Chinese grammar}

For a long time, people have focused on the dialectical analysis of the phonetic and semantic aspects of dialects, and basically formed a research method based on diachronic investigation. For the dialect grammar, there is still to be explored in the research paradigm of research methods. "Zhangjiakou Jinyu Grammar Study" draws on the subjective theory and semantic grammar of contemporary language, and makes a useful attempt in this respect. The dialect grammar of this book is divided into sections according to the national overall layout. The inductive research method shown in Figure 2 below is unique: 


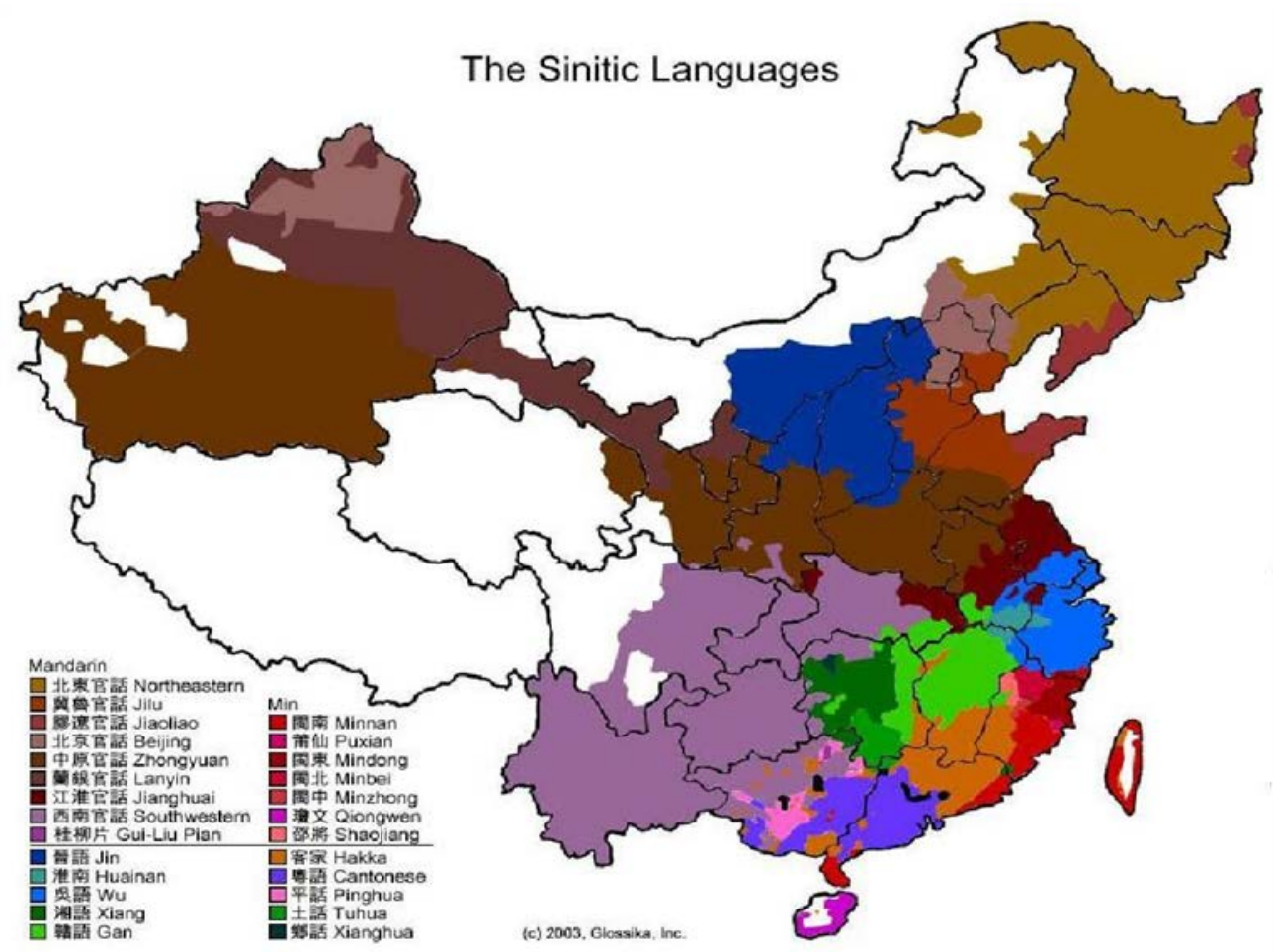

Figure 2: Distribution map of contemporary Chinese dialects

\subsection{It is a bit face-to-face, combined with deduction and deduction}

As shown in Figure 2 above, the authors based on various forms of specific corpus, inferred from the typical features, and inferred from the class features. In order to investigate the overall grammatical features of the real words and function words in Jin language, the author starts from the specific description and analysis of individual words, such as the modal verb "to wait" and the detached meaning verb "turn" as an example to describe the basic characteristics of a specific word; There is also a discussion of the commonality of a class of words, such as "diversity and type characteristics of position words", "two sets of demonstrative pronouns and combinations thereof", and the like. This combination of point and face helps to reveal the grammatical features and essential laws of Jin language from micro to macro.

\subsection{Around the grammatical form, the combination of form, function and meaning}

Because Chinese lacks rigorous morphological features like the Indo-European language family, it is necessary to take into account the form, function and meaning in grammar research. The practice of Zhangjiakou Jin grammar research shows that this point is equally important for dialect grammar research. For example, when the author examines the two-tone state words of the Lushan dialect, it is the semantic grammatical nature of the phenomenon of "derived" that is truly revealed from different angles of form, meaning and function.

\subsection{Comparison of internal and external, diachronic and synchronic "}

The father of modern linguistics" Saussure was the first to systematically propose synchronic linguistics and diachronic linguistics. Since then, according to Saussure's guidelines, linguistics, especially grammar, mainly follows the research paradigm of synchronic linguistics. Matthews of 
the Prague School and Chen Wangdao and Fang Guangtao of China were also combined with the common time, but it is still difficult to implement specific research, especially the dialect grammar. "Zhangjiakou Jinyu Grammar Study" seems to pay special attention to the combination of synchronic and diachronic research when describing each word and sentence.

For example, in the Jin language, the author points out through the diachronic comparison that the two words "one child" and "personal child" in Jin language are mainly used for objective statements, while the latter are more subjective. Another example is the overlap of Jin language. The book not only describes the "No AA" of Jin language, but also the morphological characteristics of "AA" and "Multi AA". It also compares with the historical changes of Putonghua and similar dialects.

In terms of syntactic description, the author also pays attention to revealing the motivation of sentence generation from the diachronic dimension, which enables the author to examine the essential laws of specific dialects from the two dimensions of system and historical evolution. This kind of research method not only expands the typological value of dialect grammar research, but also provides useful exploration for the combination of Mandarin and dialect in modern Chinese grammar research.

\section{The composition and breakthrough new system of contemporary Chinese grammar}

Some of the research results in "Zhangjiakou Jinyu Grammar Research" have been published in academic journals such as "Chinese Language". As a monograph, compared with the published results, this book is characterized by its strong systemicity and basically forms a new system for studying the grammar of modern Chinese dialects. This system starts from the grammatical form, from form to function and meaning, so it is structural, functional and meaningful, as shown in Table 1 below:

Table 1 Seven Architectures of Network Education Learning System

\begin{tabular}{|c|c|}
\hline Number/name & Function \\
\hline $\begin{array}{l}\text { TS130 - Student Education } \\
\text { Record (Trouse Sheet) }\end{array}$ & $\begin{array}{l}\text { Used to Transfer Student Data Between Educational } \\
\text { Institutions }\end{array}$ \\
\hline $\begin{array}{l}\text { TS131 - Student Education } \\
\text { Record (Transcript) }\end{array}$ & $\begin{array}{l}\text { Confirmation Provides intelligent automatic } \\
\text { confirmation when student records are received }\end{array}$ \\
\hline $\begin{array}{l}\text { TS146 - Student Education } \\
\text { Record (Troble Sheet) }\end{array}$ & $\begin{array}{l}\text { Allow Educational Institutions to Submit Automated } \\
\text { Student Record Requests }\end{array}$ \\
\hline $\begin{array}{l}\text { TS147 - Response to student } \\
\text { education record (transcript) }\end{array}$ & request Automated response to st \\
\hline $\begin{array}{l}\text { TS132 - Human Resource } \\
\text { Information }\end{array}$ & $\begin{array}{l}\text { Used to Transfer Personnel Record Data Between } \\
\text { Educational Agents }\end{array}$ \\
\hline 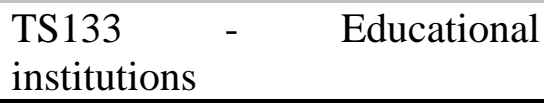 & $\begin{array}{l}\text { Used to transmit information about educational } \\
\text { institution characteristics between educational agents }\end{array}$ \\
\hline TS152 - Government Statistics & Used to send responses to government surveys. \\
\hline
\end{tabular}

In the theoretical framework shown in Table 3 above, the author's research horizons range from lexical to syntactic: the lexical part involves adverbs, prepositions, and conjunctions; the sentence part discusses declarative sentences, focus sentences, negative sentences, and interrogative sentences.

The author's description of a single word is concrete and subtle. Like the analysis of the modal verb "to wait" and the detached meaning verb "turn", all of them are small and large enough to look at the grammatical features of Jin language from individual phenomena. When examining the 
characteristics of large categories such as real words and function words, the author pays attention to communalizations, such as "diversity and type characteristics of position words" and "two sets of demonstrative pronouns and their combinations". Such a research paradigm is also applicable to the description and analysis of various sentences, thus forming a meticulous system from micro to macro, from form units to syntactic structures.

\section{Conclusion}

"Zhangjiakou Jinyu Grammar Study" reveals that we can strengthen the in-depth study of modern Chinese grammar from the perspective of dialects, which is beneficial to reveal the essence and laws of Chinese from a broader and more scientific perspective. At the same time, while drawing on the theoretical methods of contemporary linguistics, we should continue to explore new research paths based on the actual Chinese language, and truly realize the combination of diachronic and synchronic, static and dynamic, internal and external elements, and can be like Zhangjiakou Jin. The Study of Language Grammar explores boldly in new perspectives, new methods, and new systems, and finally realizes all-round breakthroughs in Chinese linguistics from theory to practice.

\section{References}

[1] Allodi M W. A Two-Level Analysis of Classroom Climate in Relation to Social Context, Group Composition, and Organization of Special Support [J]. Learning Environments Research, 2016, 5(3):253-274.

[2] Liu Y C, Wang A D, Li S G, et al. Composition and geochronology of the deep-seated xenoliths from the southeastern margin of the North China Craton[J]. Gondwana Research, 2013, 23(3):1021-1039.

[3] Girardin M P, Terrier A. Mitigating risks of future wildfires by management of the forest composition: an analysis of the offsetting potential through boreal Canada[J]. Climatic Change, 2015, 130(4):1-15. 